\title{
Confirmada la presencia y reproducción de Myotis bechsteinii en el Pirineo aragonés
}

\author{
RAmon JATO $^{1 *}$, Juan CARlos Albero ${ }^{1}$, Luis Lorente ${ }^{2}$
}

\author{
${ }^{1}$ Departamento de Especies y Hábitats. Sociedad Aragonesa de Gestión Agroambiental. \\ ${ }^{2}$ Avda Puente del Pilar 23, 6 B 50014 - Zaragoza \\ *Corresponding author e-mail: rjato@sarga.es
}

DOI: http://dx.doi.org/10.14709/BarbJ.7.1.2014.02

Spanish title: resence and reproduction of Myotis bechsteinii confirmed in the Aragonese Pyrenees

\begin{abstract}
In 2012 we began working to determine the distribution and conservation status of Myotis bechsteinii in Aragon. We surveyed 18 sites with potentially suitable habitat in the Aragonese Pyrenees and 231 individuals of 23 bat species were netted. We confirmed the presence of the Myotis bechsteinii in the Aragonese Pyrenees, providing the first data on breeding in Aragón and the northeast Iberian Peninsula. 16 individuals of Myotis bechsteinii were caught in five locations relatively close to each other. Breeding females and juveniles were captured in a single location, which is also a breeding roost located on an old specimen oak Quercus gr cerrioides with large diameter in a monospecific open woodland (dehesa), with dominance of ancient hollow trees. Counting during emergence showed that the colony was formed by 64 females and juveniles. We only trapped adult males in the other localities sample all of them within or in the vicinity of well-preserved spots Quercus gr cerrioides. The oak forests are highly degraded in Aragonese Pyrenees, with very few and small patches of healthy forest with adult specimens. This results in a very low habitats suitability for Myotis bechsteinii and a high degree of fragmentation.
\end{abstract}

Key words: Myotis bechsteinii, breeding roost, oak woodland, Pyrenees, Aragón.

Resumen: En 2012 se iniciaron los trabajos de prospección para determinar la distribución y estatus de conservación de Myotis bechsteinii en la Comunidad Autónoma de Aragón. Se muestrearon 18 localidades con hábitat potencial en el Pirineo aragonés donde se trampearon 231 ejemplares de 23 especies. Se confirma la presencia de la especie en el Pirineo aragonés y se presentan los primeros datos de reproducción en el noreste de la Península Ibérica. Se capturaron 16 individuos de Myotis bechsteinii en cinco localidades relativamente próximas entre sí. Capturamos hembras reproductoras y juveniles en una sola localidad, donde también se localizó una colonia de cría en un ejemplar de roble de Quercus gr cerrioides de gran diámetro situado en una mancha monoespecífica adehesada, con dominancia de ejemplares centenarios que mostraban abundantes oquedades. La colonia se censó en emergencia, contabilizándose 64 ejemplares entre hembras y juveniles. En el resto de localidades únicamente se trampearon machos adultos, las cuales también estuvieron situadas dentro o en las inmediaciones de manchas bien conservadas de Quercus gr cerrioides. Los robledales aragoneses del Pirineo se encuentran muy degradados, las manchas en buen estado de conservación con ejemplares adultos son muy escasas y de pequeño tamaño lo que redunda en una disponibilidad de hábitat para Myotis bechsteinii muy reducida y con un alto grado de fragmentación.

Palabras clave: Myotis bechsteinii, colonia reproductora, robledal, Pirineos, Aragón. 


\section{INTRODUCCIÓN}

En Aragón se han citado 28 especies de murciélagos, según se recoge de la información obtenida de diversos trabajos realizados entre 2001 y 2012 (Woutersen 2001 y 2004; García-González et al. 2003; Alcalde et al. 2003 y 2004; Serra-Cobo 2004; Albero et al, 2010 y 2011). La presión de muestreo en esta última década, ha permitido citar por primera vez nuevas especies y actualmente Aragón es una de las pocas comunidades autónomas con presencia de especies como Plecotus macrobullaris, Myotis cf. nattereri, y Vespertilio murinus (García-Gonzalez et al. 2003; Alcalde et al. 2008; Alberdi et al. 2009). Sin embargo, y pese a ser una comunidad con abundancia y diversidad forestal, únicamente existen tres citas históricas de presencia de Myotis bechsteinii en Aragón, dos en la provincia de Huesca (Woutersen y Bafaluy 2001) consideradas erróneas por otros autores (Alcalde et al. 2008) y otra en los Montes Universales en Teruel (Alcocer \& Monsalve, com. pers. en Alcalde et al. 2008).

En Europa M. bechsteinii aparece restringido a bosques maduros de haya y roble con una alta proporción de árboles viejos (Hutson et al. 2012; Dietz y Pir 2009). En la Península ibérica se ha comprobado que la especie se encuentra muy ligada los robledales de Quercus pyrenaica y $Q$. robur (Napal et al. 2009 y 2010, Goiti et al 2007, López y Hermida 2012), incluso en Cazorla se han encontrado ejemplares en extensos pinares (Arribazalaga et al. 2013 a y b) en los que seleccionaban los rodales de frondosas o árboles caducifolios sueltos dentro de pinares, a pesar de su baja disponibilidad mostrando una importante respuesta a variaciones a nivel de microhábitat dentro de macrohábitats aparentemente homogéneos.

La distancia de más de $200 \mathrm{~km}$. a otras poblaciones conocidas, la escasez de datos en el cuadrante nororiental de la península con unas pocas citas en Cataluña (Flaquer et al 2010), y el estado de conservación desfavorable de las masas de frondosas, especialmente de las quercíneas, hacían dudar de su presencia en el Pirineo aragonés.

M. bechsteinii es una especie prioritaria de interés europeo (Directiva 92/43/CEE) para la que las comunidades autónomas, en caso de estar presente, deben designar espacios destinados a su conservación. En base a datos erróneos y sin la constancia segura de su presencia en el Pirineo aragonés, la especie se incluyó en los inventarios de varios lugares pirenaicos de la Red Natura 2000 de Aragón. Al tratarse de una especie muy escasa, de requerimientos forestales y detección dificultosa, no suele detectarse en trabajos de inventarios generales, por lo que se ha tendido a dar por presente allí donde el tipo de hábitat así lo hacía sospechar.

En consecuencia, el Gobierno de Aragón decidió iniciar trabajos de prospección para determinar si la especie se encontraba efectivamente presente. En el presente artículo se exponen las observaciones realizadas de esta especie durante los años 2012 y 2013 en el Pirineo oscense, dentro de los trabajos de inventario de quirópteros de interés comunitario (Directiva 92/43/CEE) tanto dentro como fuera de la Red Natura 2000.

\section{MATERIAL Y MÉTODOS}

Se han prospectado 18 zonas potenciales del Pirineo de la provincia de Huesca entre el 30 de mayo y el 27 de agosto de 2012, y otra zona en 2013. La ubicación de las zonas de muestreo se realizó seleccionando masas forestales de quercíneas con presencia de pies maduros de gran tamaño (Fig. 1).

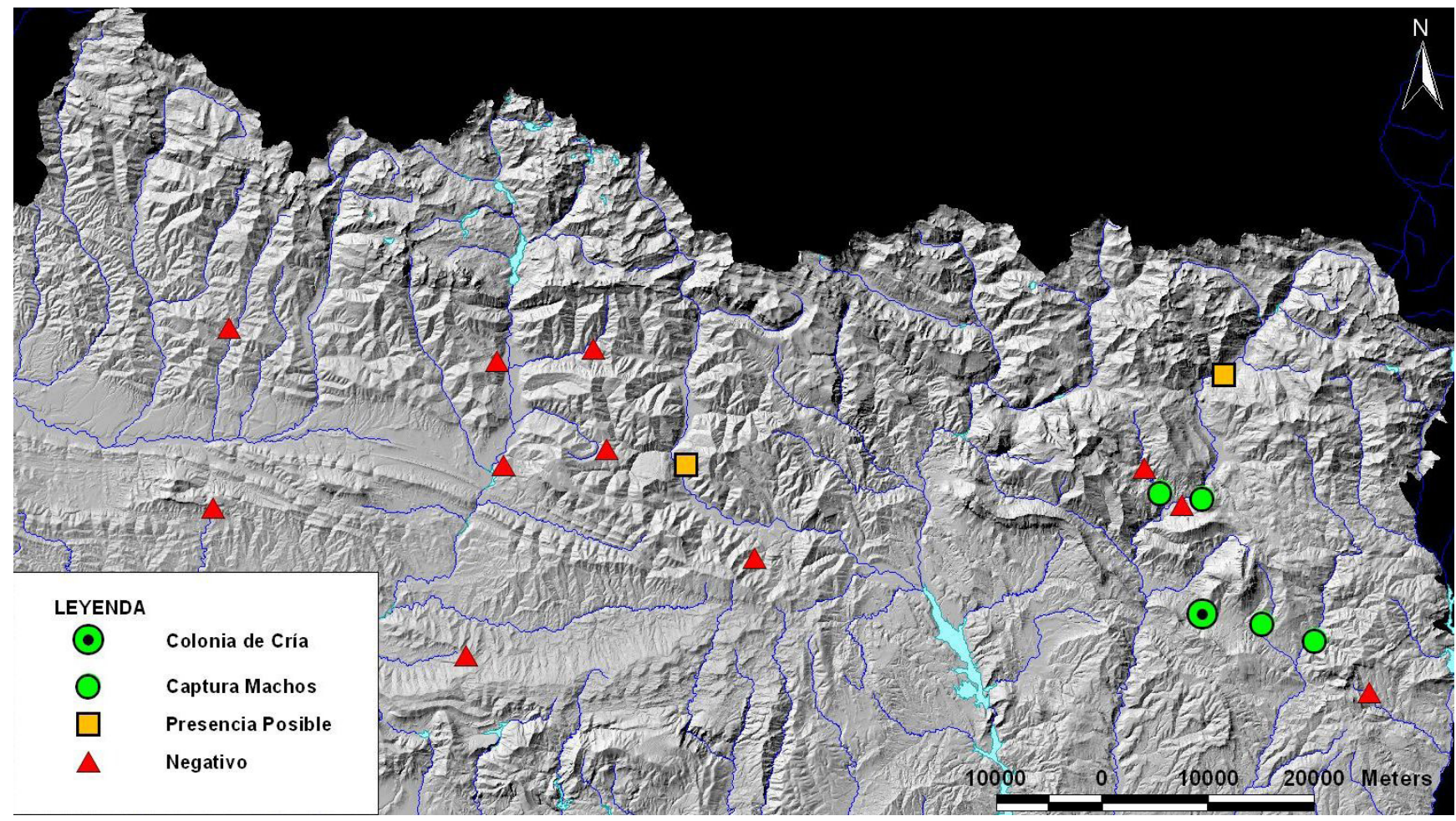

Fig. 1 - Localización de las estaciones de muestreo de Myotis bechsteinii y resultados en el Pirineo aragonés. 


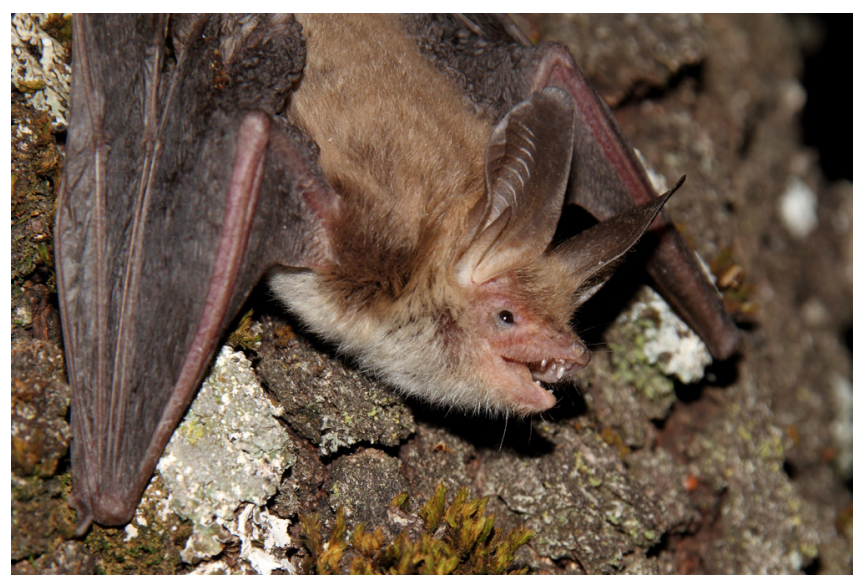

Fig. 2 - Hembra adulta de Myotis bechsteinii en un tronco de quejigo.

Para la detección de la especie se usaron tres métodos. Por un lado, se colocaron redes de niebla en puntos de agua (remansos de ríos, balsas ganaderas y depósitos). Por otro, también se instalaron redes en el interior del bosque en combinación con dos reproductores de reclamos acústicos (modelo Sussex Autobat, Universidad de Sussex). Dado el pequeño tamaño de las parcelas de bosque a muestrear optamos por un sistema intermedio entre las redes móviles usadas por Goiti et al. (2007) y las estaciones de reclamo con redes fijas, que consistió en la colocación de un número variable de redes fijas dentro del bosque moviendo los reclamos entre ellas. Por último, se realizaron grabaciones de ultrasonidos de manera irregular en bebederos con un detector D240x (Pettersson Elektronik AB, Uppsala, Suecia) y de manera regular con un equipo de detección y grabación EM3+ (WildLife Acoustic, Concord, EEUU) en modo automático tanto en bebederos como en el interior del bosque. Se eligieron los días de trampeo con las condiciones meteorológicas adecuadas, ausencia de viento y precipitaciones, y temperaturas por encima de $10^{\circ} \mathrm{C} . \mathrm{El}$ muestreo comenzó una hora antes del ocaso y persistió hasta la captura de ejemplares de $M$. bechsteinii, o bien hasta que la temperatura descendía por debajo de $10^{\circ} \mathrm{C}$.

A los ejemplares capturados (Fig. 2) se les tomaron datos biométricos y se identificaron apoyados por bibliografía especializada (Dietz y Von Helversen 2004). También se tomaron muestras de tejido (biopunch en el plagiopatagio) que fueron depositadas en el Departamento de Zoología de la Universidad del País Vasco/Euskal Herriko Unibertsitatea (UPV/EHU) y en la Estación Biológica de Doñana (CSIC).
El análisis de las grabaciones de ultrasonidos se realizó mediante el software de análisis automático Sonochiro de la empresa Biotope y el programa informático BatSound 3.31 (Pettersson Electronik AB) apoyados en los criterios de identificación publicados por Barataud y Tupinier (2012), teniendo en cuenta las dificultades de discriminar las ecolocalizaciones de algunas especies.

Así mismo, a una hembra reproductora de M. bechsteinii se le adherió un radio emisor (Fig. 3) modelo Picopip de 0,35 g (Biotrack, Dorset, RU), para localizar la colonia. Se tuvo en cuenta que el peso del emisor fuera inferior al $5 \%$ del peso del ejemplar (10,6 gramos). El conteo del número de individuos en la colonia se realizó durante la emergencia mediante una cámara de video DCR-TRV14b (Sony, Japón) con foco de luz infrarroja IRLamp6 (Wildlife Engineering, EEUU).

\section{Resultados}

En total, se capturaron 231 ejemplares de 23 especies de quirópteros en 18 localidades. Respecto a $M$. bechsteinii se capturaron 16 individuos en cinco localidades, todas relativamente próximas entre sí, situadas en la comarca de la Ribagorza, en el Pirineo Oriental aragonés (Fig. 1). En un enclave (localidad 1), se capturaron hembras reproductoras y juveniles de ambos sexos, en otras tres localidades (2, 3, 4 y 7) fueron trampeados únicamente machos adultos, y en dos localidades ( 5 y 6 ) la posible presencia de la especie se basa únicamente en grabaciones (Tabla 1).

En la localidad 1 se capturaron dos hembras lactantes el 11 de julio de 2012. Posteriormente, el 30 de julio de 2012 se capturaron nueve ejemplares más, una hembra adulta y ocho jóvenes. En las otras tres localidades se capturaron machos adultos el 17, el 20 y el 23 de julio de 2012 y el 24 de agosto de 2013. Mediante el análisis de las grabaciones de ultrasonidos se identificaron llamadas de ecolocación compatibles con Myotis bechsteinii en las localidades 1, 5 y 6.

Las capturas se realizaron dentro de manchas de Quercus gr. cerrioides o en bebederos en las inmediaciones de los mismos. Este roble es un híbrido entre Quercus humilis y Quercus faginea y presenta caracteres intermedios entre esas dos especies. Ambas especies y su híbrido son marcescentes.

Tabla 1. Resultados por localidades y métodos de muestreo de Myotis bechsteinii en el Pirineo aragonés.

\begin{tabular}{|c|c|c|c|c|}
\hline & Detector & Reclamo & Bebedero & TOTAL \\
\hline Loc. 1 ( $1^{a}$ visita $)$ & Positivo & $19 \mathrm{ad}$ & $19 \mathrm{ad}$ & 2 \\
\hline Loc. 1 ( $2^{\mathrm{a}}$ visita $)$ & Positivo & $6 \lesssim$ juv, $2+$ juv & $1+\mathrm{ad}$ & 9 \\
\hline Loc. 2 & Negativo & $1 \hat{o} \mathrm{ad}$ & Negativo & 1 \\
\hline Loc. 3 & & & $1 \lesssim \mathrm{ad}$ & 1 \\
\hline Loc. 4 & & & $1 \hat{\sigma} \mathrm{ad}$ & 1 \\
\hline Loc. 5 & Positivo & Negativo & & - \\
\hline Loc. 6 & Positivo & & & - \\
\hline Loc. 7 & & $2 \widehat{o d}$ & & 2 \\
\hline
\end{tabular}


El marcaje radiotelemétrico de la hembra capturada el 30 de julio de 2012, permitió localizar la colonia de cría en un ejemplar vivo en buen estado de Quercus gr cerrioides. El árbol tenía $100 \mathrm{~cm}$. de diámetro y estaba situado en el medio de una mancha monoespecífica adehesada de 5,8 $\mathrm{Ha}$, con presencia únicamente de ejemplares centenarios (Fig 2). El refugio se encontró en una cavidad realizada por pícidos situada en la cara inferior de una rama horizontal ubicada a $7,25 \mathrm{~m}$. de altura y orientada al sur. Se encuentra a $800 \mathrm{~m}$ de altitud y a $275 \mathrm{~m}$. de distancia de un curso permanente de agua. La mancha presenta una densidad de 8,8 árboles/ Ha. con una cobertura de copas del 20,2 \%, el sotobosque está únicamente compuesto por vegetación herbácea como consecuencia del intenso pastoreo. Alrededor de esta mancha se encuentra un pequeño cauce, vegetación arbustiva, campos de cultivo, desiertos de erosión sobre margas y manchas de roble mucho más densas con un escaso grado de desarrollo. Se contabilizaron 62 y 64 ejemplares en la colonia, los días 31 de julio y 2 de agosto de 2012 respectivamente, comprendiendo adultos y crías que ya eran capaces de volar.

La localidad 2 es una mancha de 3,18 Ha. de Quercus gr cerrioides con un escaso desarrollo del sotobosque que se encuentra representado casi exclusivamente por Buxus sempervirens. La mancha presenta un alto grado de madurez con una buena representación de todas las clases diamétricas con alta presencia de árboles de más de $40 \mathrm{~cm}$ de diámetro y algunos ejemplares de más de 80 y $100 \mathrm{~cm}$. de diámetro. La cobertura de copas es alta con presencia de pequeños claros diseminados por la mancha. El ejemplar macho adulto se capturó a $900 \mathrm{~m}$. de altitud y a $150 \mathrm{~m}$. de un curso permanente de agua.

Las localidades 3, 4 y 7 comprenden puntos de agua a una altitud de entre $800 \mathrm{~m}$. y $1.000 \mathrm{~m}$. dentro o muy cerca de manchas de $Q$. gr. cerrioides de diferentes grados de madurez, que se alternan con prados y bosquetes de coníferas. En estas localidades sólo se capturaron machos adultos de $M$. bechsteinii.

La localidad 5 son prados de siega en diferente grado de abandono situadas en fajas estrechas. En las márgenes de los prados hay una gran abundancia de robles de gran diámetro que confieren a la localidad un aspecto de bosque adehesado. La altitud es de $950 \mathrm{~m}$. y el curso de agua permanente más cercano se encuentra a $522 \mathrm{~m}$.

La localidad 6 se encuentra en la orilla de un embalse a $1.077 \mathrm{~m}$. de altitud situado junto a una gran mancha forestal mixta con quejigo, avellano y fresno como especies principales y algunas manchas de pino silvestre. Los quejigos en ocasiones forman pequeñas manchas monoespecíficas.

\section{Discusión y Conclusiones}

Se ha podido confirmar la presencia y reproducción de M. bechsteinii en el Pirineo aragonés. En la única colonia localizada, se contabilizaron en emergencia nocturna 64 ejemplares entre hembras y crías por lo que la colonia estudiada estaría en concordancia con el tamaño descrito en la literatura (entre 6 y 50 hembras adultas; Napal et al. 2009; Dietz et al. 2009). Podría considerarse como una colonia

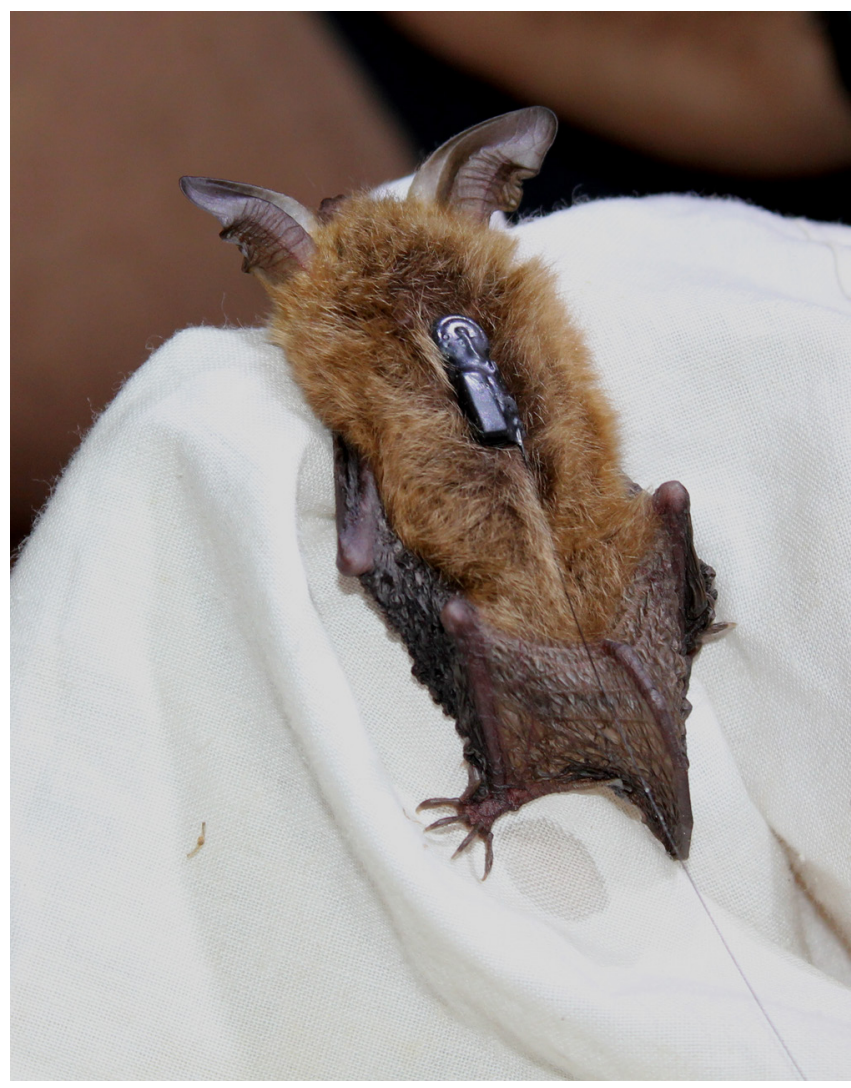

Fig. 3 - Hembra adulta de M. bechsteinii con emisor modelo Picopip de 0,35 g (Biotrack, Dorset, RU).

mediana-grande, de un mínimo de 32 hembras reproductoras estimado considerando una proporción 1:1 entre hembras y crías (Gessner y Weishaar 2004).

Los tres métodos usados en el presente estudio han arrojado resultados positivos a la hora de detectar M. bechsteinii, aunque con diferente éxito. De los puntos con resultados positivos, la especie se capturó con redes en bebedero en tres de las seis localidades en las que se colocaron; con redes y reclamo en el bosque en tres de las cuatro en las que se usó; por último, se registraron grabaciones compatibles con $M$. bechsteinii en 3 de las 4 localidades en las que se utilizó este método (Tabla 1). La dificultad para discriminar las ecolocaciones de las especies del género Myotis mediante el análisis de ultrasonidos, hace que las localidades 5 y 6 se deban considerar únicamente como posibles. Además, el uso de este método sólo es recomendable como complementario a las capturas con red, aunque puede ser útil para realizar prospecciones previas y centrar los esfuerzos de captura en las localidades en las que se realicen grabaciones compatibles con los parámetros de los ultrasonidos emitidos por la especie.

El uso de reclamos parece favorecer la captura de $M$. bechsteinii con redes de niebla dentro del bosque (hembras lactantes y posteriormente crías dependientes en localidad 1, un macho en localidad 2, y dos machos en la localidad 7), aunque es evidente que el uso de redes colocadas en bebederos situados cerca de hábitats favorables también ha resultado efectivo con capturas de hembras reproductoras en la localidad 1, y de machos en las localidades 3 y 4 .

La colonia de cría se encuentra en una mancha forestal adehesada rodeada de desierto de erosión, campos de cultivo y manchas de bosque muy joven (Fig. 3). Tanto la colonia de 


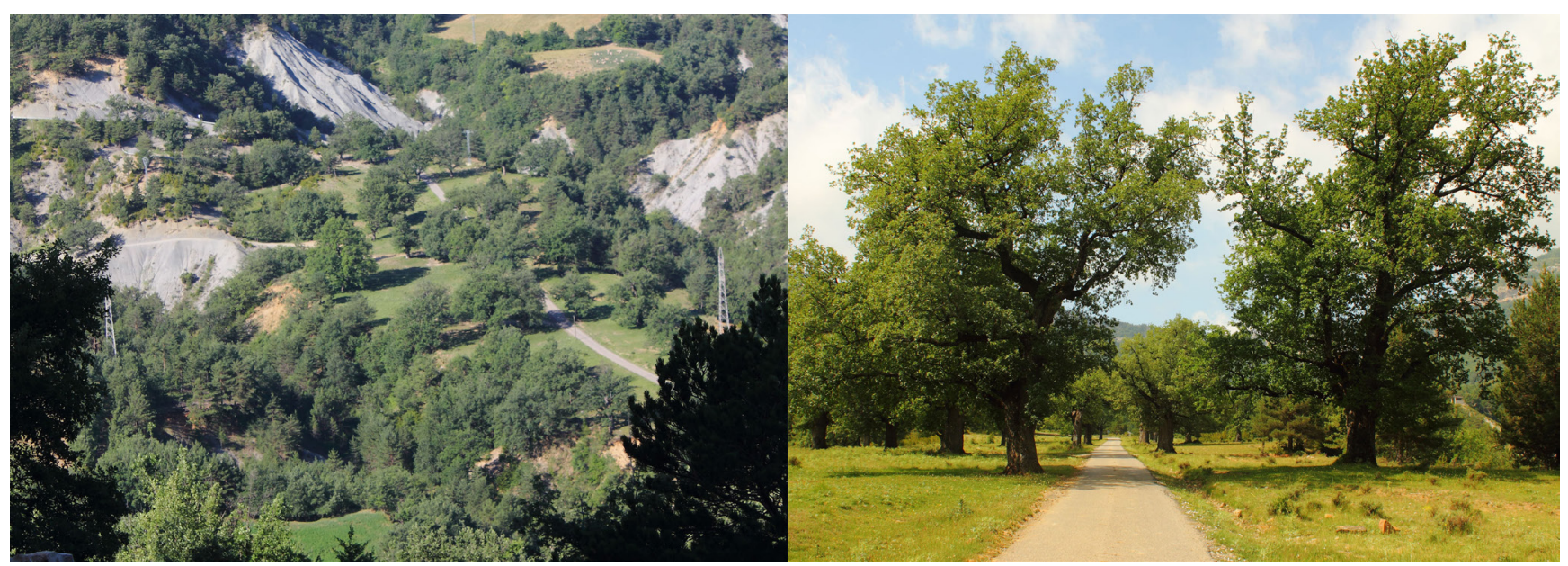

Fig. 4 - Vistas general y de detalle de la zona en la que se encuentra la primera colonia hallada de Myotis bechsteinii en el Pirineo aragonés.

cría como su entorno más inmediato no se corresponden con los óptimos descritos en otras poblaciones (Dietz y Pir 2009; Napal et al. 2009 y 2010), aunque sí coincide con lo descrito en Kan̆uch y Cel'uch (2007) donde encuentran en un bosque adehesado poblaciones de $M$. bechsteinii asociadas con otras especies forestales.

Las masas de quercíneas del Pirineo aragonés, compuestas por las especies Quercus ilex y $Q$. gr cerrioides, fueron intensamente aprovechadas para la obtención de leñas y producción de carbón por lo que hoy en día solo se encuentran masas degradadas en proceso de regeneración, con gran abundancia de pies formados a partir de rebrotes vegetativos. Los bosques maduros son casi inexistentes y sólo perviven pequeñas manchas con presencia de ejemplares con un mínimo de madurez. Las zonas con presencia de ejemplares añosos se encuentran normalmente ligadas a pequeñas manchas adehesadas en las que se ha realizado un aprovechamiento tradicional de los pastos, también a agrupaciones de ejemplares trasmochados de gran tamaño, así como a alineaciones de ejemplares que sobrevivieron gracias a su uso como puntos de referencia para delimitar propiedades. Algunas de estas manchas permanecen aisladas o bien diferenciadas de otros hábitats forestales, mientras que en otras ocasiones han quedado inmersas dentro de extensas masas forestales con un escaso grado de madurez general.

Todo parece indicar que solamente en alguna de estas pequeñas zonas es donde pueden encontrarse las últimas colonias del Pirineo aragonés. En concordancia con los resultados de Arribazalaga et al. (2012 y 2013) y Napal et al (2013) parece que Myotis bechsteinii podría estar utilizando en Aragón pequeños parches de hábitat favorable dentro de grandes extensiones con hábitat poco adecuado; incluso que a pesar de ser una especie más abundante en el contexto centroeuropeo que en el área mediterránea podría tener su óptimo ecológico en los bosques templados mediterráneos y que su escasez en la biorregión estaría reflejando el alto grado de fraccionamiento y degradación de los bosques caducifolios mediterráneos (Napal et al, 2013).

De las cinco localidades con resultados positivos, dos de ellas (entre las que se encuentra la única colonia de cría conocida de Aragón) se encuentran fuera de la Red Natura 2000, de las otras 3 dos se encuentran en LIC y la última en una
ZEPA. El alto grado de fragmentación y el pequeño tamaño de las manchas de hábitat potencial hace que la idoneidad del hábitat deba de ser detectada a nivel de microhábitat lo que ha propiciado que las parcelas con hábitat potencial para la especie no hayan sido incluidas en la Red Natura 2000 más que por mero azar o debido a encontrarse dentro de grandes masas forestales que por su extensión han sido expresamente seleccionadas. Para garantizar la protección y gestión adecuada de la especie y sus hábitats es importante la localización de las colonias en futuras prospecciones, la identificación de las manchas de quercíneas con abundancia de pies maduros y su inclusión en la Red Natura 2000.

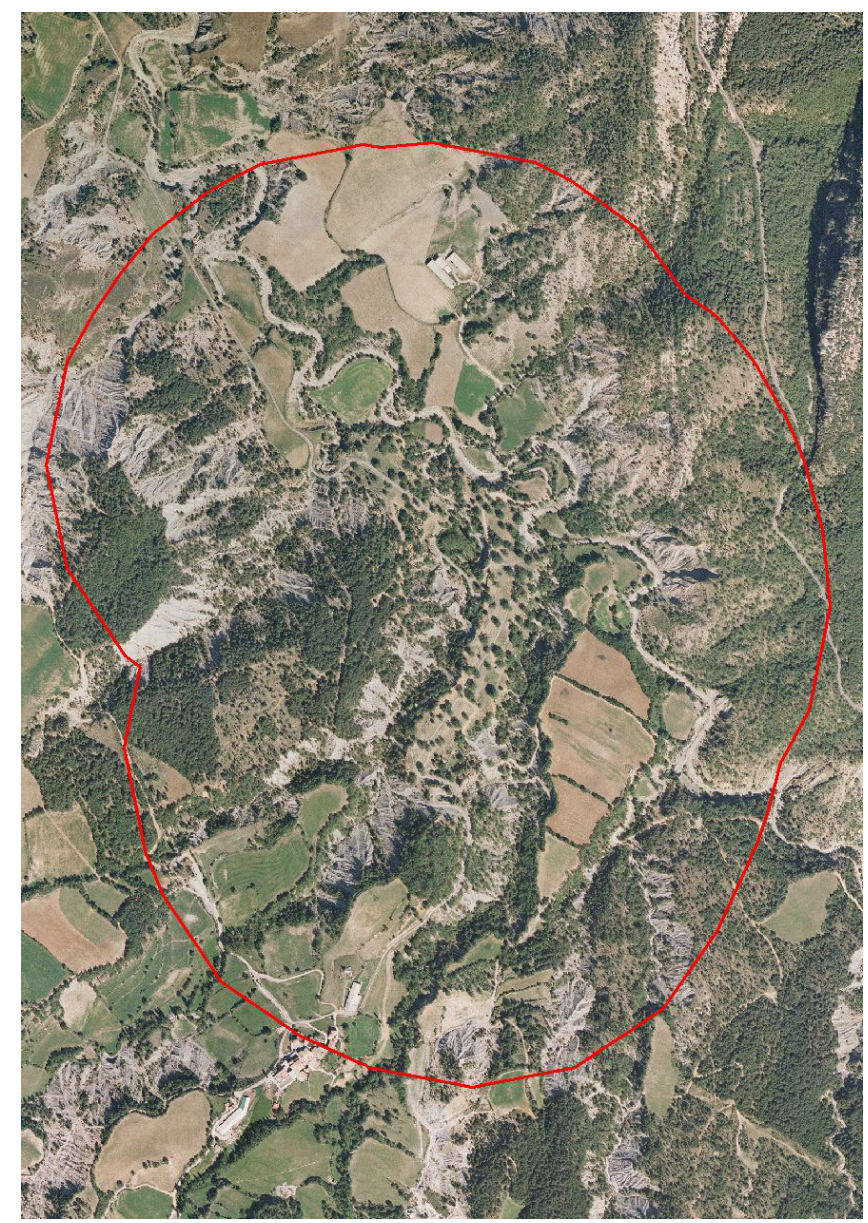

Fig. 5 - Vista aérea de la mancha en la que se encuentra la colonia de Myotis bechteinii con buffer de $500 \mathrm{~m}$. 


\section{Agradecimientos}

Este proyecto ha sido financiado por el Servicio de Biodiversidad de la Dirección General de Conservación del Medio Natural del Gobierno de Aragón, bajo la dirección de Manuel Alcántara.

Inazio Garin y Jose Ramón Aihartza (UPV/EHU), Javier Juste (EBD-CSIC) y Xavier Puig (Associació Galanthus) nos prestaron los equipos de reclamo Autobat, nos asesoraron sobre su uso y compartieron su valiosa experiencia sobre la especie. Los Agentes de Protección de la Naturaleza del Gobierno de Aragón (APN) Mariano Muñoz y Félix Calvo nos ayudaron a localizar manchas forestales bien conservadas. Nos han acompañado en el trabajo de campo Merichel Broto, Fernando Carmena, Ignacio Gómez, Jesús Laín, Quintina Ortiz, Javier Miranda y Juan Ramón Donés de la empresa SARGA, y Félix Calvo APN del Gobierno de Aragón. A Begoña Marquina por la revisión del resumen en inglés. A Inazio Garin por la revisión del manuscrito y sus valiosas aportaciones.

\section{REFERENCIAS}

Alberdi, A., Aihartza, J., Albero, J. C., Aizpurua, O., López-Baucells, A., Freixas, L. \& Garin, I. (2012). First records of the parti-coloured bat Vespertilio murinus (Chiroptera: Vespertilionidae) in the Pyrenees. Mammalia, 76(1), 109-111. DOI: http:// dx.doi.org/10.1515/MAMM.2011.106

Albero J.C., Ballarín I., Carmena F., Rivas J.L., Sanz J. \& HernándeZ D. 2010. Inventario de refugios y especies de quirópteros en el Parque Natural de la Sierra y Cañones de Guara. SODEMASA. Gobierno de Aragón. Informe inédito.

Albero J.C., Broto M., Carmena F., Gómez I., Jato R., Rivas J.L., \& SANZ J. 2011. Inventario de refugios y especies de quirópteros en el Parque Natural de Posets-Maladeta. SODEMASA. Gobierno de Aragón. Informe inédito.

Alcalde, J.T., Trujillo, D., Artárcoz, A. \& Agirre-Mendi, P.T., 2008. Distribución y estado de conservación de los quirópteros en Aragón. Graellsia, 64(1), 3-16.

Amengual B., Aymerich P., Fontal J., Gosalbez J., Götzens G., López M. \& Serra - Cobo J. 2004. Estado de conservación de las poblaciones de quirópteros, mamíferos acuáticos y Rana pyrenaica del Parque Nacional de Ordesa y Monte Perdido. Propuestas para su eficaz gestión. Areambiental 2004. Ministerio de Medio Ambiente. PNOMP. Informe Técnico. DOI: http://dx.doi.org/10.3989/graellsia.2008.v64.i1.51

Arribazalaga, A., Saldamendi, E., Napal, M., Garín, I. Aimartza, J. 2013. Selección del hábitat por Myotis bechsteinii en un pinar al sureste de la Península Ibérica: cuando el macrohábitat enmascara al microhábitat. Barbastella, 6 (1), 95.
Barataud, M. \& Tupinier, Y. 2012. Écologie acoustique des chiroptères d'Europe: identification des espèces, étude de leurs habitats et comportements de chasse. Ed Biotope.

CArro, F. 2007. Myotis bechsteinii (Kuhl, 1817). [Ficha de Atlas]. Pp. 171 -173 en L. J. Palomo, J. Gisbert \& J. C. BLANCO (eds.): Atlas y Libro Rojo de los mamíferos terrestres de España. MMA/DGCN-TRAGSASECEM-SECEMU, Madrid.

Dietz, C. \& Von Helversen, O. 2004. Claves de identificación de los murciélagos de Europa. Publicación electrónica.

DiETz, M. \& PIR, J. B. 2009. Distribution and habitat selection of Myotis bechsteinii in Luxembourg: implications for forest management and conservation. Folia Zoologica, 58(3), 327-340.

Diet, C. Helversen, O. \& Nill, D. 2009. Bats of Britain, Europe and Northwestern Africa. A \&C Black Publisher Ltd. London.

Fitzsimons, P., Hill, D. \& Greenaway, F. 2002. Patterns of habitat use by female Bechstein's bats (Myotis bechsteinii) from a maternity colony in a British woodland. Report. School of Biological Sciences, University of Sussex.

Flaquer, C., Torre, I. \& Arrizabalaga, A. 2007. Selección de refugios, gestión forestal y conservación de quirópteros forestales. Conservación de la biodiversidad, fauna vertebrada y gestión forestal. J.Camprodon y E. Plana (eds). Publicacions i Edicions de la Universitat de Barcelona, 2007.

Flaquer C., Puig X., Fábregas E., Guixé D., Torre I., RÀFols R.G., PÁramo F., CAMProdon J., Cumplido J.M., Ruíz-Jarillo R., Baucells A.L., FreiXas L. \& Arrizabalaga, A. 2010. Revisión y aportación de datos sobre quirópteros de Catalunya: propuesta de lista roja. Galemys, 22 (1): 29-61.

García-GonzÁlez, R., Aihartza, J.A., Garín, I., Goiti, U., Trujillo, D., Zabala, J., Gosalbez, J., LuQue, J.J., Monreal, E., Aymerich, P., Götzens, G., Couto, S., Herrero, J., Hernández, Y. \& Prada, C. 2003. Inventario y criterios de gestión de los mamíferos del PNOMP, OAPN - CSIC, Huesca, Jaca.

Gessner, B. \& WeishaAR, M. 2004. Fort setzung der Beringung der Bechsteinfledermaus (Myotis bechsteinii) in der Region Trier = Continuing the ringing of Bechstein's bats (Myotis bechsteinii) in the region of Trier. Dendrocopos, 31, 21-26.

Goiti, U., Aihartza, J., Garin, I. \& SAlsamendi, E. 2007. Surveying for the rare Bechstein's bat (Myotis bechsteinii) in northern Iberian peninsula by means of an acoustic lure. Hystrix, the Italian Journal of Mammalogy, 18(2), 215-223. DOI: http://dx.doi. org/10.4404/hystrix-18.2-440 
Hohti, P., Celuch, M., DAnko, Š., \& KaňUCh, P. 2010. Constraints in the roost-site selection of the treedwelling Bechstein's bat (Myotis bechsteinii). Hystrix, the Italian Journal of Mammalogy, 22(1).

Hutson,A.M.,Spitzenberger,F., Tsytsulina,K.,Aulagnier, S., Juste, J., Karataş, A., Palmeirim, J. \& Paunović, M. 2008. Myotis bechsteinii. In: IUCN 2012. IUCN Red List of Threatened Species. Version 2012.2. $<\underline{w w w . i u c n r e d l i s t . o r g}>$. Downloaded on 16 April 2013

KAŇUCH, P., \& CEL'UCH, M. 2007. Bat assemblage of an old pastured oak woodland (Gavurky Protected Area, central Slovakia). Vespertilio, 11: 57-64.

Kerth, G., WAGNer, M. \& KöNIG, B. 2001. Roosting together, foraging apart: information transfer about food is unlikely to explain sociality in female Bechstein's bats (Myotis bechsteinii). Behavioral Ecology and Sociobiology, 50(3), 283-291. DOI: http://dx.doi. org/10.1007/s002650100352

Kerth, G. \& Morf, L. 2004. Behavioural and genetic data suggest that Bechstein's bats predominantly mate outside the breeding habitat. Ethology, 110(12), 987-999. DOI: http://dx.doi.org/10.1111/j.1439$\underline{0310.2004 .01040 . x}$

Kerth, G. \& Petit, E. 2005. Colonization and dispersal in a social species, the Bechstein's bat (Myotis bechsteinii). Molecular Ecology, 14(13), 3943-3950.

LóPEZ, Z. y HermidA, R.X. 2013. Estudio del uso del hábitat por Myotis bechsteinii en los montes de O Morrazo (Pontevedra). Barbastella, 6 (1) 95-96.
Napal, M., Garin, I. , Goiti, U., Salsamendi, E. y Aihartza, J., 2009. Selection of maternity roosts by Myotis bechsteinii (Kuhl, 1817) in the Southwestern Iberian Peninsula. Acta Chiropterologica, 11(2), 425-433.

Napal, M., Garin, I., Goiti, U., Salsamendi, E. \& Aihartza, J. 2010. Habitat selection by Myotis bechsteinii in the southwestern Iberian Peninsula. Annales Zoologici Fennici, 47(4), 239-250. DOI: http://dx.doi. org/10.5735/086.047.0402

NAPAl, M., Garin, I., Goiti, U., SAlsamendi, E., \& Aihartza, J. (2013). Past deforestation of Mediterranean Europe explains the present distribution of the strict forest dweller Myotis bechsteinii. Forest Ecology and Management, 293, 161-170. DOI: http://dx.doi. org/10.1016/j.foreco.2012.12.038

RecKardt, K. \& Kerth, G. 2007. Roost selection and roost switching of female Bechstein's bats (Myotis bechsteinii) as a strategy of parasite avoidance. Oecologia, 154(3), 581-588. DOI: http://dx.doi. org/10.1007/s00442-007-0843-7

Salsamendi, E., NAPAl, M.,Aihartza, J., Goiti, U.,Almenar, D. \& GARIN, I. 2007. Estudios de seleccion de hábitat en Myotis bechsteinii, Myotis emarginatus y Rhinolophus euryale. Informe final. SECEMU, Junta de Extremadura.

Woutersen, K. \& Bafaluy J.J. 2001. Murciélagos del Alto Aragón. Kees Woutersen Publicaciones, Huesca. 